\title{
Sistema de monitoreo remoto para manejo integrado de plagas en cultivos de Chile Habanero
}

\section{Remote monitoring system for integrated pest management on Habanero Crops}

DURÁN-LUGO, Juan Miguel†*, QUEJ-COSGAYA, Héctor Emmanuel, CUEVAS-HERNÁNDEZ, Omar Yared y QUEJ-GARCÍA, Emmanuel Guadalupe

Universidad Autónoma de Campeche

ID $1^{\text {er }}$ Autor: Juan Miguel, Durán-Lugo / ORC ID: 0000-0001-7179-4301, CVU CONACYT ID: 653065

ID 1 ${ }^{\text {er }}$ Coautor: Héctor Emmanuel, Quej-Cosgaya / ORC ID: 0000-0001-5603-3037, CVU CONACYT ID: 204225

ID $2^{\text {do }}$ Coautor: Omar Yared, Cuevas-Hernández / ORC ID: 0000-0001-6264-4838, CVU CONACYT ID: 999933

ID $3^{\text {er }}$ Coautor: Emmanuel Guadalupe, Quej-García / ORC ID: 0000-0001-5044-680X, CVU CONACYT ID: 974716

DOI: $10.35429 / J U S D .2019 .16 .5 .24 .38$

Recibido: 30 de Junio, 2019; Aceptado 30 Septiembre, 2019

\begin{abstract}
Resumen
Plagas, enfermedades y cambios climatológicos provocan pérdidas hasta por un $50 \%$ en cultivos de Chile Habanero, mediante el desarrollo e implementación del Sistema de Monitoreo Predictivo Remoto de Plagas en Cultivos de Chile Habanero, consistente en la instalación de una estructura capaz de integrar sensores de temperatura, humedad, lluvia, etc. alimentado mediante energía solar en los centros de cultivo experimental, se trasmite la información por Radio Frecuencia y GSM hasta el centro de monitoreo, el cual cuenta con un software predictivo que genera el porcentaje de ataque de las plagas comunes que inciden en plantas de acuerdo al sensado en el campo de cultivo, posteriormente concluye con recomendaciones específicas para el control biológico y/o químico pertinente. Con el apoyo en validación experimental por dos Centros de Investigación Agrícola y una asociación de agricultores, se experimentó en tres campos de cultivo, disminuyendo las pérdidas del $40 \%$ al $68 \%$ por concepto de plagas. Finalmente, con un costo de inversión del $1.4 \%$ del monto total de las pérdidas económicas por productor, se alcanzaron ganancias extras de entre $\$ 136,000$ a $\$ 165,000$ por ciclo de cultivo en terrenos de 4 hectáreas, implementando el sistema y siguiendo las recomendaciones predictivas del mismo.
\end{abstract}

Telemonitoreo, Lógica difusa, Agroindustria

\begin{abstract}
At least 50\% of Habanero crop losses are caused by pests, diseases and climatological changes. The crop requires development and implementation of a tool called Remote Predictive Monitoring System for pest on Habanero crops, this consists of installing a structure that is able to integrate sensors that measure temperature, rain, humidity,etc. The system is powered by solar energy at experimental crop centres. The data is sent by radio frequency and GSM to the monitoring centre, where a predictive software generates a percentage of common pest attacks that affect the plants included in the crop field sensed. Finally the system provides specific recommendations for biological and chemical control. With the validation of the experiment by two Farm Research Centres and a Farmers Association, the system was installed on three experimental crop fields, reducing crop losses from $40 \%$ to $68 \%$. With an investment of $1.4 \%$ (equivalent to the money lost per crop), implementing the system and following the recommendations it was achieved that there would be extra profits between $\$ 136,000$ and $\$ 165,000$ per crop cycle of 4 hectares. This project is being considered for patent registry as a prototype to replicate for other crops.
\end{abstract}

Telemonitoring, Fuzzy logic, Agroindustry

Citación: DURÁN-LUGO, Juan Miguel, QUEJ-COSGAYA, Héctor Emmanuel, CUEVAS-HERNÁNDEZ, Omar Yared y QUEJ-GARCÍA, Emmanuel Guadalupe. Sistema de monitoreo remoto para manejo integrado de plagas en cultivos de Chile Habanero. Revista del Desarrollo Urbano y Sustentable. 2019. 5-16: 24-38

\footnotetext{
* Correspondencia del Autor (jumduran@uacam.mx)

$\dagger$ Investigador contribuyendo como primer Autor
} 


\section{Introducción}

Mediante el acercamiento al sector agrícola de nuestra nación, se identificaron las diferentes problemáticas que existen en la planeación y cultivo de diversas especies de plantas comestibles y derivados, originado de esto y planeando la aplicación de sistemas ingenieriles del área de mecatrónica, se procedió a investigar diferentes alternativas innovadoras a los métodos tradicionalistas empleados en los diferentes esquemas de producción agrícola. Un gran desafío para los biotecnólogos involucrados en el área de la agricultura es satisfacer la demanda cada vez mayor de una población en constante crecimiento se estima que para el 2050 habrá una población de 9 mil millones de personas que demandarán alimentos (Godfray, 2010).

En específico la producción de chiles en México varía entre 870,000 y 1,400,000 toneladas, que se obtienen en una superficie que oscila entre 92,000 y 110,000 hectáreas. Esto ubica a nuestro país como el tercer productor mundial y exportador, el 5.8\% de su producción lo exporta a países como Estados Unidos, Canadá, Alemania, España y Suecia, entre otros.

La producción de chile habanero en México (SAGARPA, 2018) se ubica en los estados de Campeche, Quintana Roo, Yucatán, Tabasco, Chiapas, Veracruz, Zacatecas, Coahuila, Aguascalientes, Jalisco y Michoacán. Los rendimientos son muy variables, siendo en los estados de Jalisco y Campeche donde se obtienen los mayores rendimientos (40-50 y 30 toneladas por hectárea, respectivamente).

El consumo de chiles (ajíes picantes) es muy amplio y popular en México, donde, de acuerdo con Gómez y Schwentesius (1995), se encuentra la mayor variedad genética del género Capsicum del mundo. Sin embargo, el chile habanero (Capsicum Chinense) es originario de Sudamérica, aunque también es conocido en el sureste de México, especialmente en Yucatán, pues ha formado parte de la mundialmente reconocida gastronomía yucateca.
En el mundo el chile habanero es uno de los de mayor pungencia o picor (registra de 200 000 a 500000 unidades "Scoville") por su alto contenido de capsaicina, por lo que es muy apreciado e igualmente debido a su capsaicina para la elaboración de cosméticos, pomadas "calientes", gas lacrimógeno, recubrimiento de sistemas de riego para protección de roedores y pintura para barcos por su alta capacidad anticorrosiva (Ledón, 2001).

\section{Planteamiento del problema}

La producción media de una hectárea de Chile Habanero es de 10 toneladas y requiere una inversión aproximada de 64 mil pesos (INIFAP, 2017); tomándose en cuenta el costo a la compra de entre $\$ 18$ - $\$ 20$, el monto recaudado por hectárea en un ciclo de cosecha es de $\$ 180,000$ a $\$ 200,000$. A su vez cada productor en promedio cuenta con 4 hectáreas de siembra lo que en total representaría un costo de inversión de $\$ 256,000$ con un monto de venta de entre $\$ 720,000$ y $\$ 800,000$ pesos, teniendo ganancias en un rango de $\$ 464,000$ a $\$ 544,000$ pesos.

Las plagas, enfermedades, cambios climatológicos y variaciones del precio en el mercado, provocan pérdidas por más del $50 \%$ en cultivos de Chile Habanero lo que representa un monto aproximado de $\$ 360,000$ a $\$ 400,000$; lo que finalmente impacta en el margen de ganancia del productor alcanzando un monto máximo de $\$ 144,000$ pesos. El campo mexicano se enfrenta a grandes desafíos: tener disponibilidad de agua, aumentar la producción, al mismo tiempo que se reduce el uso de fertilizantes químicos y erradican melazas agresivas, para ello, tiene que hacer uso de la biotecnología, que es un recurso tecnológico que permite reforzar las acciones de trabajo para mantener en ascenso la producción agrícola (SAGARPA, 2017).

El constante desarrollo de los sensores y protocolos de comunicación remotos para la observación del planeta tierra, ha generado una gran cantidad de información útil para estudiar y monitorear de una mejor forma recursos naturales tales como suelo, agua y clima, permitiendo resolver problemas de escases de alimentos, contaminación del medio ambiente, manejo ecológico de recursos naturales y el estudio del crecimiento acelerado de áreas urbanas, (Díaz- Padilla et al., 2001). 
De acuerdo con Diaz-Padilla et al. (2001), el ejemplo primordial de clasificación de las agro potencialidades son los requerimientos agroecológicos de los cultivos, esto implica conocer cuáles son las necesidades de temperatura, precipitación, altitud, pendiente, suelos, etc. Y encontrar en qué zonas o regiones cumplen dichos requerimientos, en forma óptima, mediana, baja o no apta.

Entre los beneficios que aporta la biotecnología se encuentran: generar productos de mayor calidad a bajo costo, así como reducir el impacto negativo en el medio ambiente. Esta importante área se apoya en tecnológicas y estrategias complementarias, como lo es la Ingeniería Mecatrónica con una línea de generación y adquisición de tecnología enfocada al área Agroindustrial.

\section{Objetivos}

\section{Objetivo General:}

Reducir las pérdidas por concepto de enfermedades en cultivos de Chile Habanero (Capsicum Chinense), desarrollando e implementado un sistema predictivo de monitoreo remoto para el manejo sustentable de plagas.

\section{Objetivos Específicos:}

- Predecir mediante lógica difusa la aparición de las principales plagas que atacan cultivos de chile habanero, previniendo de tal modo las enfermedades en los cultivos.

Generar un sistema de monitoreo remoto, integrando una Interfaz Gráfica de Usuario implementada en una Web Page amigable a la vista e intuitiva.

Brindar a los productores una guía estratégica de control sustentable de plagas mediante agentes biológicos y/o químicos, ajustable a sus necesidades específicas.

- $\quad$ Reducir el uso de agroquímicos en los campos de cultivo.

\section{Marco Teórico}

\section{Chile Habanero}

El chile habanero es originario de América del Sur, aunque también es ampliamente conocido en el sureste mexicano donde forma parte de la gastronomía regional. El chile habanero es uno de los de mayor pungencia o picor en el mundo, su contenido de capsaicina es entre las 200,000 a 500,000 unidades "Scoville" (Bosland, 1996; Long-Solís,1998; Ramírez et al., 2005). Esa cantidad de capsaicina ha sido determinante en el incremento de la demanda de esta especie de chile en el mercado nacional e internacional. $\mathrm{La}$ capsaicina tiene amplia utilización en la medicina, cosméticos, pinturas, gases lacrimógenos y salsas (Soria et al., 2002; Salazar et al., 2004). En México, los estados que producen el chile habanero son Tabasco, Campeche, Quintana Roo, Sonora, Veracruz, Chiapas y Baja California Sur. La mayor superficie cultivada se encuentra en el estado de Yucatán con un 73\% (708.43 ha) del total de la superficie sembrada (SIAP-SAGARPA, 2007).

El cultivo de chile habanero, bajo condiciones de campo, no se lleva a cabo en forma comercial en las regiones áridas del norte de México. Esto debido a que las altas temperaturas e incidencia solar presentes hacen que la planta tenga un desarrollo raquítico y una baja producción lo cual lo hace incosteable. Sin embargo, el chile habanero es un cultivo atractivo ya que su precio en el mercado nacional supera a la de cualquier otro tipo de chile, además el chile habanero es un producto que tiene demanda a nivel nacional e internacional por sus múltiples usos.

El chile habanero muestra su mejor desarrollo en zonas templadas, subtropicales. Con altitudes que oscilan entre 0 y $2700 \mathrm{msnm}$. Se desarrolla en un rango de precipitación óptima de 600 a $1250 \mathrm{~mm}$ (FAO, 2014). Sin embargo, estos valores varían en base a la variedad que se vaya a cultivar y la adaptabilidad que ésta presenta (FAO, 2014; Aragón, 1995). El chile habanero es una hortaliza de clima caliente, los rangos de temperatura en que se desarrolla de forma normal son: mínima $10^{\circ} \mathrm{C}$, máxima $35^{\circ} \mathrm{C}$ y óptima de $30^{\circ} \mathrm{C}$. Las temperaturas menores de $10^{\circ} \mathrm{C}$ y mayores a $35^{\circ} \mathrm{C}$ limitan el desarrollo del cultivo (Ramírez et al., 2006). La temperatura para la germinación fluctúa entre los 18 y 35 ${ }^{\circ} \mathrm{C}$, siendo la óptima de $30^{\circ} \mathrm{C}$.

DURÁN-LUGO, Juan Miguel, QUEJ-COSGAYA, Héctor Emmanuel, CUEVAS-HERNÁNDEZ, Omar Yared y QUEJ-GARCÍA, Emmanuel Guadalupe. Sistema de monitoreo remoto para manejo integrado de plagas en cultivos de Chile Habanero. Revista del Desarrollo Urbano y Sustentable. 2019. 
Los suelos más favorables para el desarrollo del chile habanero son aquellos que tienen buen drenaje y buena retención de humedad. Con un $\mathrm{pH}$ de 6.5 a 7.0, para lograr una mayor disponibilidad de los nutrientes; $\mathrm{pH}$ del suelo diferentes a estos valores necesitarán enmiendas por lo que es muy importante conocer y considerar este factor para el buen uso de fertilización y asimilación de los nutrientes. El cultivo de chile habanero requiere una lámina de riego de 750 a $1000 \mathrm{~mm}$ para obtener altos rendimientos. Una lámina de riego menor a $30 \mathrm{~mm}$ mensuales afecta el rendimiento, el cual se ven disminuido (Ramírez et al., 2006).

Existen más de 200 variedades de chile habanero (Figura1), sin embargo, en México y en especial en la Península de Yucatán se cultivan únicamente tres variedades: Kukulkán, Chichén Itzá y Jaguar (SAGARPA, 2016).

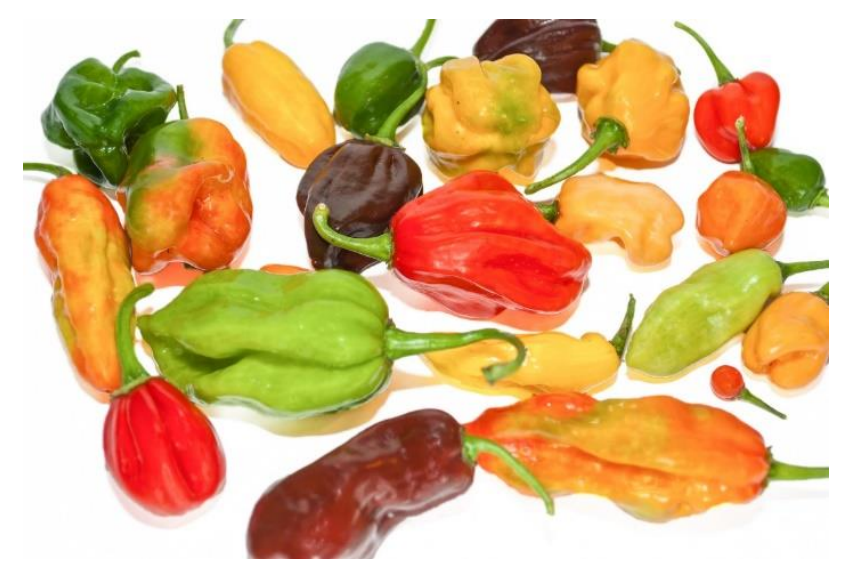

Figura 1 Variedades de Chile habanero

Fuente: www.ethno-botanik.org, 2019

Las tres presentan buen desarrollo vegetativo con una altura promedio de $1 \mathrm{mt}$ en campo abierto y $2.1 \mathrm{mt}$ en casa sombra. La variedad Kukulkán presenta mayor rendimiento por hectárea con 18 mil 760 kilogramos; seguida por la variedad Chichén Itzá, con 17 mil $67 \mathrm{~kg} / \mathrm{ha}$; y por la variedad Jaguar, con 16 mil $389 \mathrm{~kg} / \mathrm{ha}$ de rendimiento. Finalmente, en cuanto a calidad del fruto, la variedad Jaguar obtuvo un mayor porcentaje de frutos de primera calidad, con $88.81 \%$, seguido de la variedad Kukulkán, con $78.44 \%$ y Chichén Itzá con $75.23 \%$. Por tal motivo los productores de la región seleccionan de forma predominante cultivar de la variedad Kukulkán, ya que presenta mayores rendimientos y en cuanto a calidad se encuentra en segunda posición.

\section{Manejo Integral de Plagas}

El Manejo Sustentable de Plagas (MSP) o Manejo Integrado de Plagas (MIP) tienen por objetivo mantener a las poblaciones plaga bajo el nivel de daño económico, protegiendo la salud humana y el medio ambiente, para lo cual utiliza todas las herramientas de control disponibles para el control de plagas (ver Figura 2).

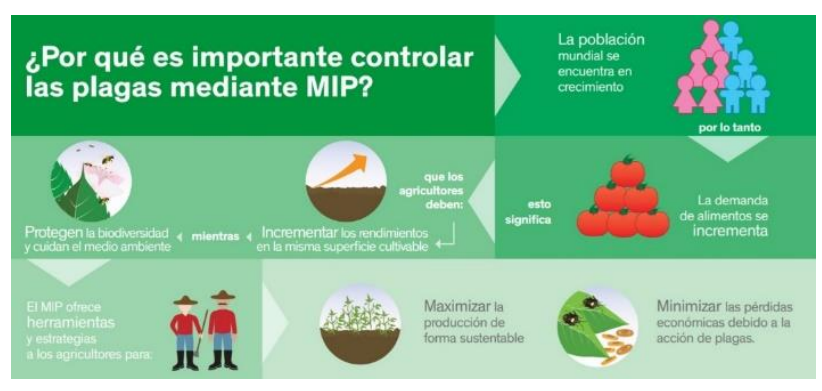

Figura 2 Importancia del MIP

Fuente: Folleto Dow AgroSciences, 2019.

Existen tres pasos que se deben tener en cuenta: prevención, observación e intervención (ver Figura 3).

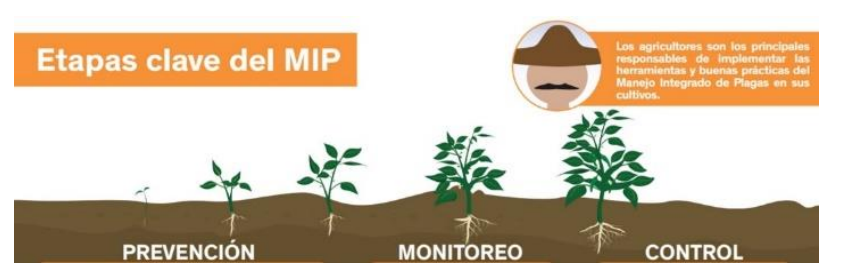

Figura 3. Etapas clave del MIP

Fuente: Folleto Dow AgroSciences, 2019.

\section{Prevención}

Limitar o prevenir las plagas, manejando el cultivo de manera de aumentar las poblaciones de enemigos naturales, disminuyendo los sitios o nichos de distintas plagas y/o disminuyendo alimento para las plagas. Para romper el ciclo de la plaga algunas herramientas a usar son: rotación de cultivos, variedades resistentes de plagas, buena sanidad, remoción de plagas y hospederos, manejo de restos de cosecha, rastrojos y semillas. También existen métodos espaciales, de secuencia y de control de material de plantación o siembra. Métodos espaciales: como, por ejemplo, usar varios patrones de cultivos, espaciamiento de plantas, cultivos intercalados, cultivos en hileras, uso de cultivo trampa o intercalados con otros, manejo del hábitat. Métodos secuenciales: como, por ejemplo, rotación de cultivos, cultivos múltiples, cultivos entre otros o bajo otro. 
Control de material de plantación/siembra: como, por ejemplo, la resistencia de planta hospedera, uso de semillas y plantas libres de enfermedades, diversidad genética del cultivo, fertilización y riego apropiado, etc.

\section{Observación}

El objetivo de este aspecto de la protección de los cultivos es el de determinar qué medida tomar y cuándo tomarla. Monitoreo del cultivo: La inspección de los cultivos a intervalos regulares es un aspecto clave. Hay que averiguar cómo crecen los cultivos, las malezas, insectos y enfermedades que están apareciendo, para llegar a una decisión en cuanto al uso de fertilizantes, control de malezas, de insectos y enfermedades y finalmente la fecha en que la cosecha debe empezar.

También hay que evaluar los enemigos naturales, ya que su presencia puede permitir que se minimice el uso de productos fitosanitarios. Se debe contar el número de plagas presentes y, frente a umbrales conocidos y el número de enemigos naturales presentes en el cultivo, se puede llegar a una decisión en cuanto a las acciones apropiadas. Sistemas de apoyo de las decisiones: A fin de ayudar a los agricultores a tomar decisiones en cuanto a la incidencia de plagas en sus campos o predios, se hacen investigaciones para determinar en qué punto deben ser realizadas ciertas acciones. Por ejemplo: cuando la población de un insecto dañino llega a un nivel determinado en las plantas cultivadas, podría ser recomendado el tratamiento con un insecticida.

Tal recomendación se haría de acuerdo a la fase de crecimiento del cultivo y la presencia de insectos benéficos. Asimismo, es posible que asesores de organismos públicos u otros tengan programas de previsión para dar consejos a los agricultores acerca del momento de emprender actividades de control de las plagas. Manejo regional: Para permitir el control eficaz de ciertas plagas, es posible que haya que tomar medidas de control de gran envergadura, sobre todo cuando se trata de plagas muy móviles. En estos casos, probablemente todos los agricultores en una localidad determinada deban realizar las acciones apropiadas.
Habitualmente tal acción coordinada sería organizada por organismos públicos. Por ejemplo, el manejo de mosca blanca.

\section{Intervención}

El objeto de las medidas de intervención directa es reducir las poblaciones de plagas a niveles económicamente aceptables. Los tipos de control son: Químico: con sustancias orgánicas e inorgánicas. Pueden ser sintéticas, organismos o derivados de organismos (biopesticidas, feromonas, aleloquímicos, reguladores de crecimiento de insectos) o provenientes de recursos naturales (inorgánicos). Biológico: la intervención biológica utiliza a organismos predadores, parasitoides o patógenos de plagas. Estos pueden ser introducidos directamente. Cultural: son medidas de manejo, tradicionales o no, que pueden ser preventivas o intervencionistas.

La manera de actuar es haciendo inaceptable la planta para la plaga, adecuando el establecimiento de la planta de estación o espacio o haciendo que el cultivo sea peligroso para la plaga por los tamaños poblacionales de enemigos naturales. En esta categoría se encuentran, por ejemplo, la rotación de cultivos, los cultivos intercalados, los cultivos trampa, el uso de plantas y/o semillas certificadas, la siembra y cultivo en época adecuada, el manejo de riego y fertilización apropiados, etc. Físico: los manejos físicos pueden alterar las características físicas del ambiente para manejar las poblaciones plaga.

Entre estos figuran, por ejemplo, la destrucción de residuos de cosecha, el laboreo del suelo apropiado, las barreras físicas como invernaderos y mallas, la solarización, el manejo del nivel de humedad del suelo para manejar algunas plagas, etc. Genético: control a través de manejo de genes, cromosomas y sistemas reproductivos de cultivos, plagas y poblaciones benéficas. Ejemplos: resistencia de planta hospedera, esterilización de insectos machos, mejora genética de enemigos naturales, etc. 


\section{Plagas y enfermedades comunes en cultivos de Chile Habanero}

La baja producción de Chile Habanero debido a la poca tecnología en el cultivo, semilla y control de plagas, ha sido un detonante de múltiples estudios de los factores que impiden el buen término de ciclo de cosecha. De acuerdo a la Secretaria de Agricultura, Ganadería, Desarrollo Rural, Pesca y Alimentación (SAGARPA) y al Instituto Nacional de Investigaciones Forestales, Agrícolas y Pecuarias (INIFAP), en su Agenda Técnica Agrícola 2015 para el Paquete Tecnológico del chile habanero, plantea tres estrategias en su cultivo:

- Controlar las plagas: Las principales plagas son picudo del chile, mosca blanca, minador de la hoja, acaro, araña, etc.

- Controlar las enfermedades: Bacteriosis, virosis y nematodos.

Control de malezas.

En el caso de control de plagas existen tres estrategias a seguir: control preventivo y técnicas culturales, control biológico y control químico (como se menciona en el apartado 4.2). Para el caso de las enfermedades, es necesario erradicar primeramente la plaga, para posteriormente aplicar estrategias en el alivio de los síntomas. Finalmente, el control de malezas debe ser constante en el cultivo ya que son la principal fuente de plagas y las plagas de las enfermedades, por lo tanto, el acabar o controlar las malas hierbas y restos de cultivo potencializan la salud de nuestras plantas, previniendo la infestación de plagas y la proliferación de las enfermedades que impactan en la perdida de la producción del cultivo.

\section{Diseño metodológico}

Mediante la implementación de un sistema sustentable de monitoreo predictivo remoto para plagas en cultivos de Chile Habanero se desarrolló un prototipo tecnológico. Para el desarrollo de este fue necesario subdividirlo en siete etapas: diseño de estructura, sistema de monitoreo in situ, sistema de telecomunicación (RF-GSM), receptor/gestor de datos (respectivamente), modelo predictivo por lógica difusa, Interfaz Gráfica de Usuario (GUI) montada en una página Web y evaluación en un campo de cultivo.

\section{Diseño de estructura soporte}

En esta primera etapa, se procedió a dibujar la estructura que va a contener los diferentes sensores, mediante el software llamado SolidWorks se definieron cinco niveles, como se aprecia en la Figura 4, con la finalidad de que cada sensor pueda ser ajustable a la etapa de ciclo de la planta que corresponda, teniendo en cuenta que en específico la planta chile habanero puede llegar hasta los $1.5 \mathrm{mts}$ (en cielo abierto) y $2 \mathrm{mts}$ en invernadero (Bogeret et al, 2010).

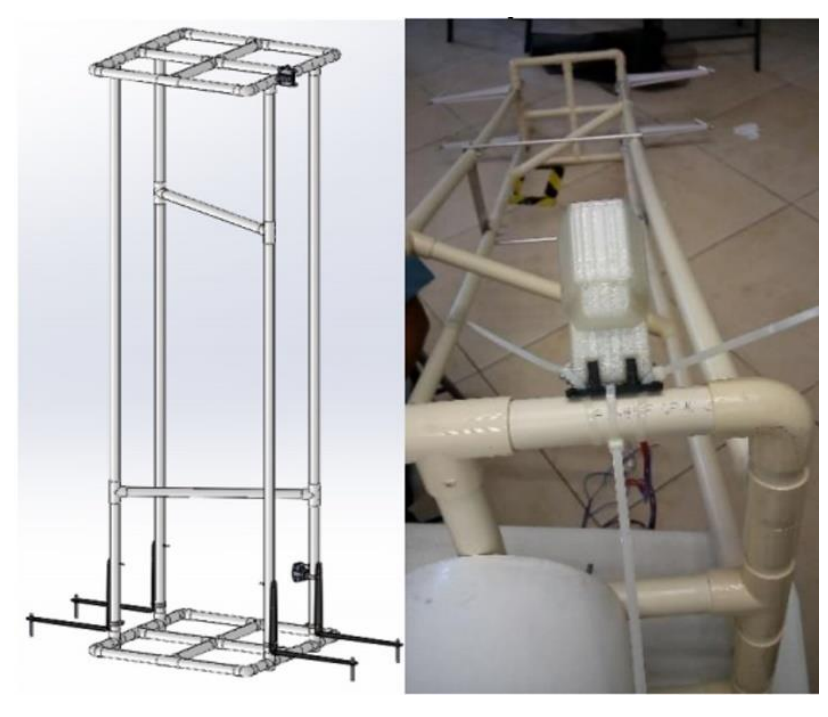

Figura 4. Estructura soporte

Fuente: Elaboración Propia, 2019

Teniendo en cuenta el clima de la región, así como la geolocalización de los campos de prueba la estructura fue diseñada de un material llamado Policloruro de Vinilo Clorado (CPVC), el cual es un termoplástico altamente recomendado en aplicaciones industriales debido a sus características de resistencia química y altas temperaturas originadas por radiación solar. Para dicha estructura, fue prevista una zona de colocación bajo tierra $(25 \mathrm{~cm})$ y una altura total de la estructura sobre tierra de $2 \mathrm{mts}$.

De igual manera se diseñó e imprimió en 3D con un material denominado PETG (Gricol de Tereftalato de Polietileno) resistente a radiación solar, las bases adaptables de cada sensor, así como del panel fotovoltaico $\mathrm{y}$ soporte de batería (ver Figura 5). 


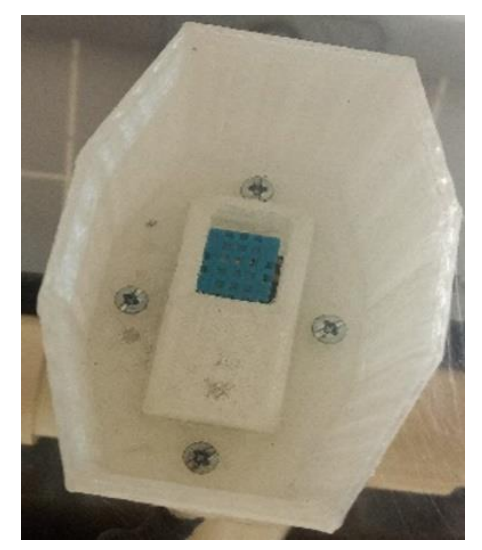

Figura 5 Encapsulados PETG impresos en 3D Fuente: Elaboración Propia, 2019

\section{Sistema de sensado in situ}

Para la medición de variables de cultivo, de acuerdo a la bibliografía revisada, se definió la necesidad de ubicar cuatro sensores en sitios estratégicos de la estructura, como se muestra en la Figura 6.

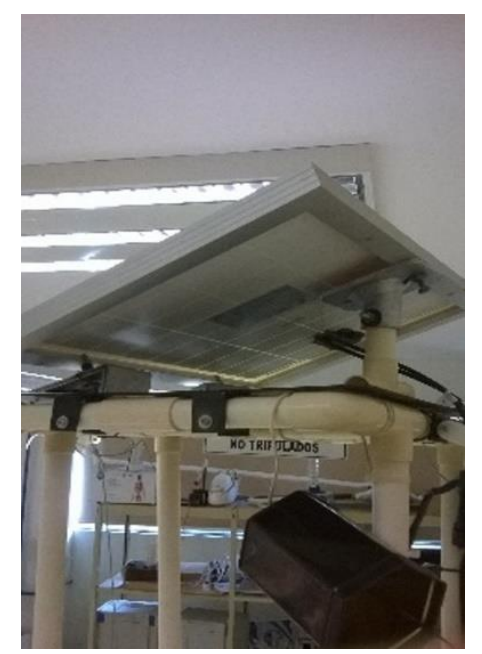

Figura 6 Colocación de sensores en estructura Fuente: Elaboración Propia, 2019

Sensor de Temperatura para subsuelo DSB18B20 (Figura 7): Ubicado a $25 \mathrm{~cm}$ bajo la superficie de la tierra, este sensor configurado de forma analógica es idóneo cuando queremos medir la temperatura en ambientes húmedos e incluso dentro del agua, aunado a su forma de sonda impermeable. Su alimentación es en el rango de $3 \mathrm{~V}-$ $5.5 \mathrm{~V}$, logrando medir temperaturas de $55^{\circ} \mathrm{C}$ hasta $125^{\circ} \mathrm{C}$.

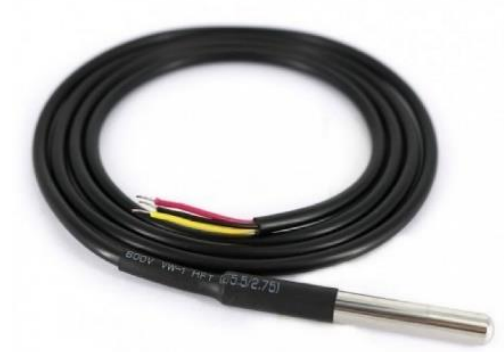

Figura 7 Sensor de temperatura subsuelo Elaboración Propia, 2019

\begin{abstract}
Sensor de Temperatura / Humedad inferior de planta DHT11 (Figura 8) Ubicado $10 \mathrm{~cm}$ sobre el nivel del suelo (altura recomendada), ajustable en un rango de $0 \mathrm{~cm}-1 \mathrm{mt}$ sobre el nivel del suelo, configurado de forma analógica ideal en la medición de temperatura y humedad en rango de $0^{\circ} \mathrm{C}-50^{\circ} \mathrm{C}$ y $20 \%$ - $90 \%$ respectivamente. Alimentado con $5 \mathrm{VCD}$.
\end{abstract}

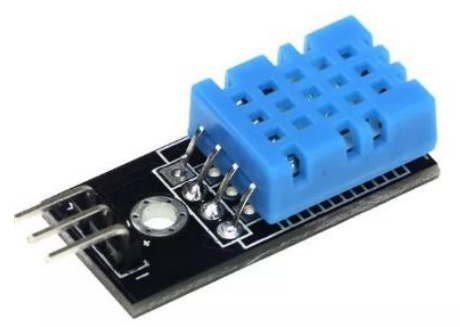

Figura 8 Sensores de temperatura / humedad inferior y superior de la planta

Fuente: Elaboración Propia, 2019

- Sensor de Temperatura / Humedad superior de planta DHT11: Sensor idéntico al mencionado anterior, definido para la medición por arriba de la planta, ajustable desde $10 \mathrm{~cm}$ hasta $2 \mathrm{mts}$ de la estructura. Su colocación dependerá del ciclo de crecimiento de la planta y se deberá ajustar de forma manual.

- $\quad$ Sensor de lluvia ARD-355 (Figura 9): Ubicado en la parte superior de la estructura con una inclinación de $45^{\circ}$, alimentado por $5 \mathrm{VCD}$, suministra una señal analógica de conductividad eléctrica en un rango de 0-5 VCD. 


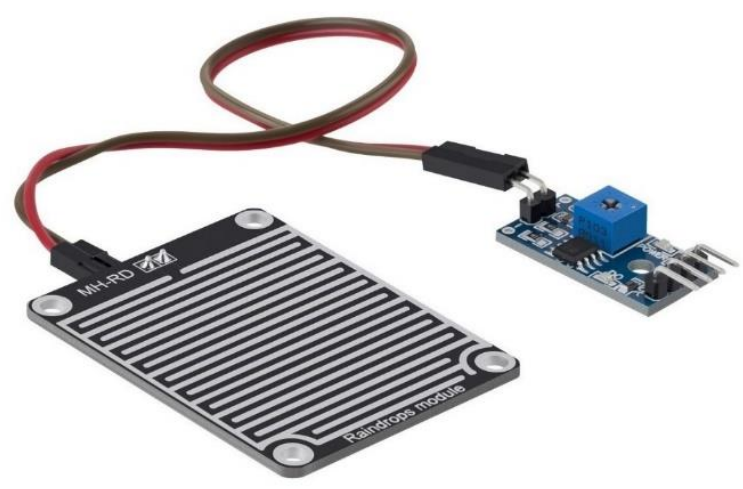

Figura 9 Sensor de lluvia

Fuente: Elaboración Propia, 2019

\section{Sistema de telecomunicaciones $\mathbf{T x} / \mathbf{R x}$}

El sistema de telecomunicación, resultó ser posiblemente el mayor de los desafíos al igual que el de suministro de energía, lo anterior debido a la ubicación geográfica de los campos de cultivo. El $80 \%$ de la producción de Chile Habanero, se encuentra en zonas remotas, en donde la inexistencia de señal celular, u algún otro medio de comunicación dificulta la transferencia de información de un punto a otro, en especial para el caso del proyecto que busca que el usuario tenga una interacción en tiempo real con los datos medidos del cultivo.

Para tal efecto, se decidió usar tecnología RF (Radio Frecuencia), la cual tiene alcance de hasta un radio de $1 \mathrm{~km}$ a una velocidad de transferencia de 2Mbits por segundo, ideal para la implementación de sistemas IoT (Internet de las Cosas). Específicamente de acuerdo a sus especificaciones y flexibilidad en el escalamiento del proyecto los dispositivos implementados son:

\begin{abstract}
Módulo NRF24L01 (Figura 10): Implementado de forma unidireccional Tx, alimentado a 5VCD con acoplador de voltaje, protocolo SPI (Serial Peripheral Interface), distancia máxima de transmisión a 700 mts óptimo, a $2.4 \mathrm{GHz}$ de banda ISM (Industrial, Scientific and Medical) para uso no comercial.
\end{abstract}

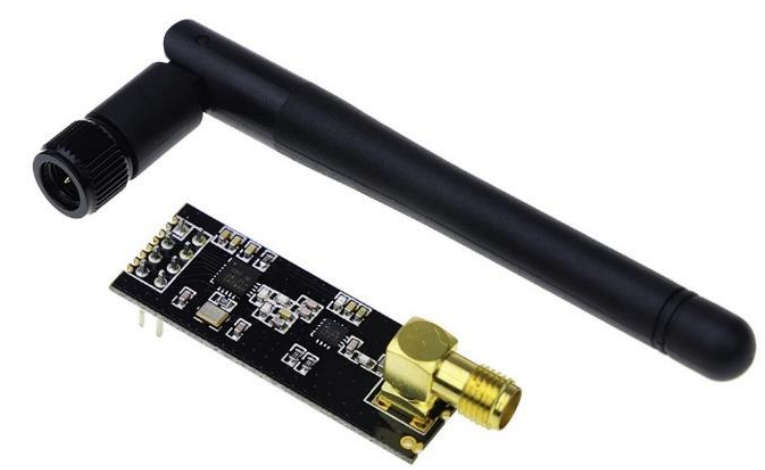

Figura 10 Módulo RF Tx/Rx

Fuente: Elaboración Propia, 2019

Módulo NRF24L01 (Figura 10): Implementadode forma unidireccional Tx, alimentado a 5VCD con acoplador de voltaje, protocolo SPI (Serial Peripheral Interface), distancia máxima de transmisión a $700 \mathrm{mts}$ óptimo, a $2.4 \mathrm{GHz}$ de banda ISM (Industrial, Scientific and Medical) para uso no comercial.

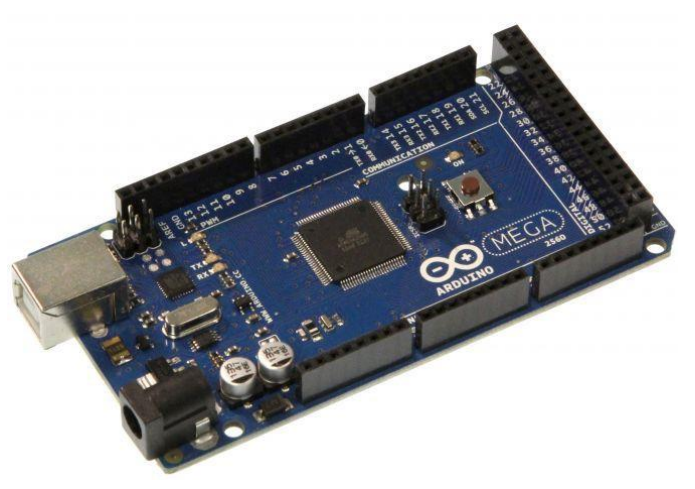

Figura 11 Microcontrolador

Fuente: Elaboración Propia, 2019

Finalmente, todo el sistema cableado e implementado en una placa PCB, fue colocado en el interior de una caja tipo gabinete Nema en la parte media de la estructura, colocando la antena en la parte exterior evitando de tal manera la disminución de su rango de operación debido a obstáculos en su dispersión de señal (Figura 12). 


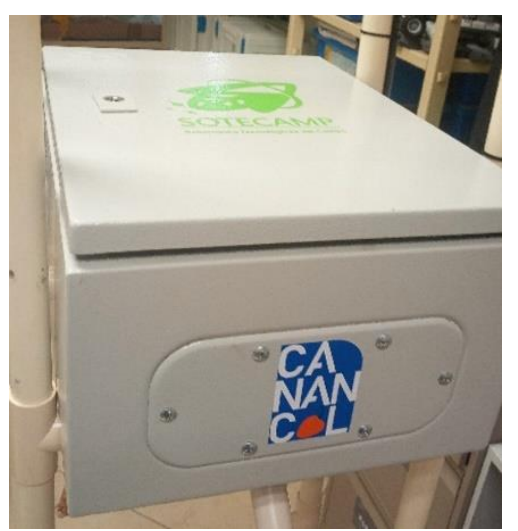

Figura 12 Encapsulado final de circuito en sistema in situ Fuente: Elaboración Propia, 2019

Es importante señalar que debido a las condiciones de humedad de la región fue necesario colocar en el interior del contenedor, un recipiente deshumificador recargable de Cloruro de Calcio, con la finalidad de proteger los componentes electrónicos de algún tipo de daño por exceso de humedad.

\section{Suministro y adecuación de energía eléctrica}

La determinación del suministro y adecuación de energía eléctrica al igual que la telecomunicación, fueron factores concluyentes en el presente proyecto, originado de la falta en algunos predios de suministro eléctrico o de la distancia de este con respecto al campo de cultivo, fue requerido caracterizar y diseñar un sistema de suministro y adecuación de energía específico para el proyecto.

Mediante la tecnología fotovoltaica se logra la captación de energía solar necesaria para ser suministrada a nuestro sistema, de igual manera mediante baterías, se almacena la energía requerida para su uso en periodos de sombra o noche. Finalmente se requiere de una etapa de potencia capaz de adecuar el suministro eléctrico a nuestras necesidades, con la consigna del uso óptimo de energía. Para la presente etapa se implementaron los siguientes componentes:

- $\quad$ Captación de energía solar (Figura 13): Panel fotovoltaico de 25 Watts, 18.3 volts, 1.37 amperes, Voc 21.2VCD, Isc 14.44A aislado e impermeable.
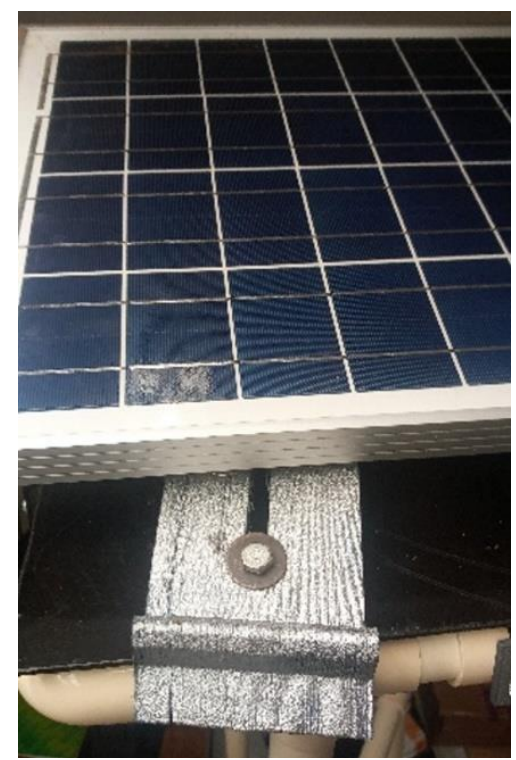

Figura 13 Panel solar

Fuente: Elaboración Propia, 2019

Almacenamiento de energía (Figura 14): Batería sellada libre de mantenimiento Osonix de $9 \mathrm{Ah}$ a $12 \mathrm{VCD}$.

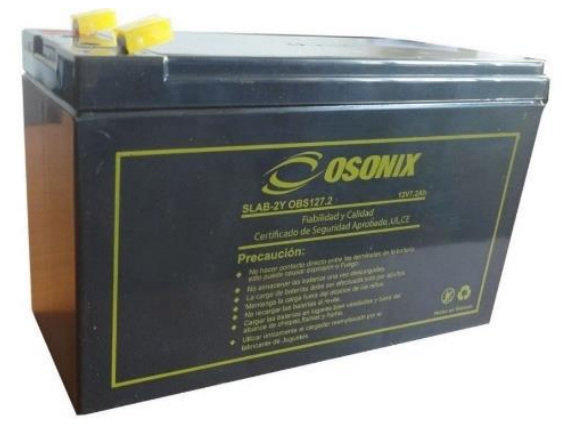

Figura 14 Almacenamiento de energía Fuente: Elaboración Propia, 2019

- Adecuación de energía para el sistema (Figura 15): Controlador de carga solar de 24VCD a 50A, pantalla LCD, conexión USB y cargador de batería PWM.

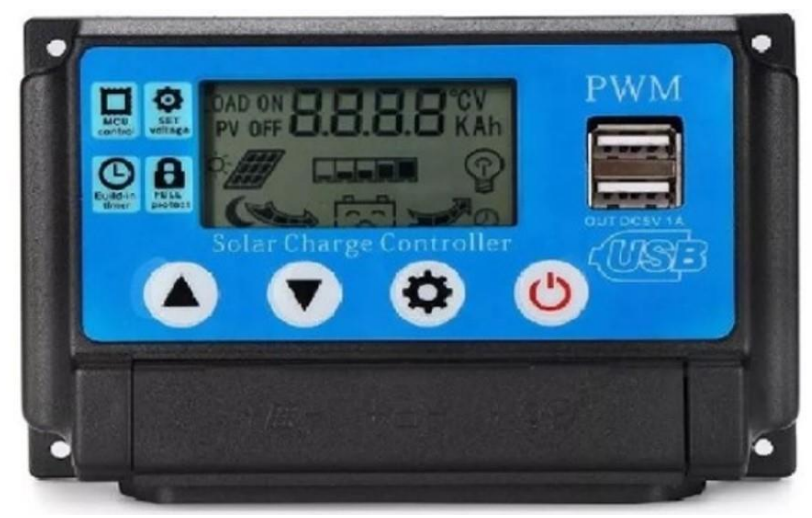

Figura 15 Adecuación de suministro eléctrico Fuente: Elaboración Propia, 2019 


\section{Sistema de telecomunicación GSM}

Esta etapa no se encuentra en condiciones de campo abierto (Figura 16), por lo que resulta relativamente sencilla su implementación, sin embargo, la implementación del protocolo y sincronización con la PC en aspectos de programación, concluyo en cierta complejidad para su interacción con la toma de decisiones e interfaz gráfica de usuario. Los componentes usados en la presente etapa fueron el Módulo GSM GPRS SIM900 configurado como Tx y el Microcontrolador ARDUINO MEGA 2560R3.

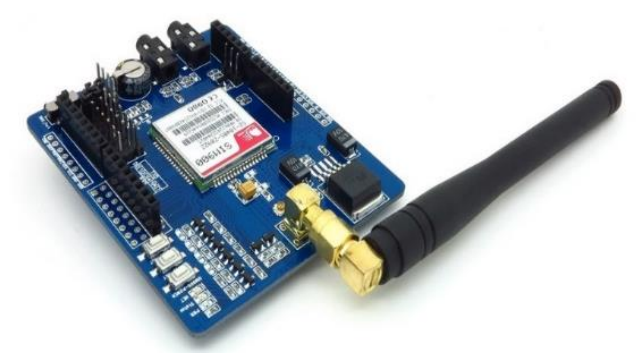

Figura 16 Módulo GSM GPRS SIM900

Fuente: Elaboración Propia, 2019

Al igual que las primeras etapas, se diseñó e imprimió en 3D una caja o chasis para almacenar los componentes electrónicos mencionados anteriormente, dejando expuesta la antena de comunicación, la entrada USB para el enlace con la PC y la entrada de alimentación de voltaje de la fuente de poder.

\section{Interfaz gráfica de usuario en Web Page}

Se diseñó una interfaz intuitiva para el despliegue de datos en una página Web, en ella se observa el porcentaje de ataque potencial de las plagas en cultivos de Chile Habanero. Los datos en la página son actualizados cada $4 \mathrm{hrs,}$ esto con la finalidad de no saturar la base de datos y brindarle al microprocesador tiempo para el procesamiento de estos en el sistema inteligente. Finalmente, el usuario puede seleccionar la plaga potencial para observar una guía con información referente a método de control biológico y/o químico, así como una breve descripción de la plaga. Para tal caso es importante resaltar que no se pretende sustituir la expertise de los agrónomos o gente dedicada al campo, sino más bien ser un complemento de estos.

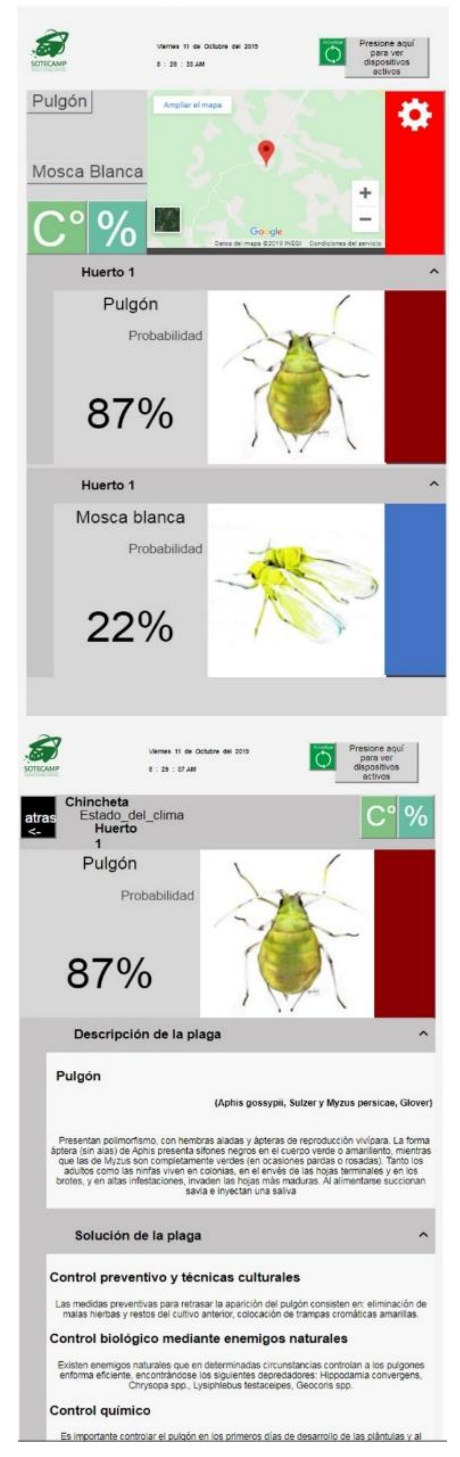

Figura 17 GUI en página Web

Fuente: Elaboración Propia, 2019

\section{Modelo predictivo}

Se definen 6 variables de entrada, siendo estas: temperatura subsuelo, temperatura inferior de la planta, humedad inferior de la planta, temperatura superior de la planta, humedad superior de la planta y porcentaje de lluvia. En el caso de variables de salida se contemplaron tres plagas con su porcentaje de aparición dependiente a las variables de entrada, siendo estas: pulgón, araña, gusano, minador de la hoja y mosca blanca.

Seguidamente se delimitaron para cada variable sus rangos de operación, subconjuntos difusos y variables lingüísticas. Por tal motivo para la variable temperatura se contemplaron las siguientes variables lingüísticas: baja, media baja, media, media alta y alta, mientras que para humedad: baja, media y alta. 
Para las variables de salida y con la finalidad de brindarle al usuario un resultado lo más intuitivo posible solo se contemplan tres: baja probabilidad, media probabilidad y alta probabilidad.

Seguidamente se definen de acuerdo a la bibliografía y a la recolección de información mediante el Método de Entrevistas realizadas a agricultores e investigadores las reglas de inferencia difusas. Teniendo en cuenta que se delimitaron 6 variables de entrada $(3$ con 5 subconjuntos cada una y 2 con 3 subconjuntos cada uno), así como 6 variables de salida con 3 subconjuntos cada una, tenemos por lo tanto un total de 21 variables de entrada y 15 variables de salida a inferir, lo que se traduce en $2^{\wedge} 21=$ $2,097,152$ posibles combinaciones.

Finalmente, al probar el sistema de fusificación mediante el Método de Mamdani y debido al alto número de inferencias difusas, se obtiene una gráfica con cambios sutiles y precisos, pero con un tiempo de procesamiento aproximado a 2 segundos (elevado para un procesamiento de datos por software). Este último factor es despreciable ya que no es requerido tomar acciones en tiempo real, por lo que es preferible adoptar precisión y sacrificar velocidad.

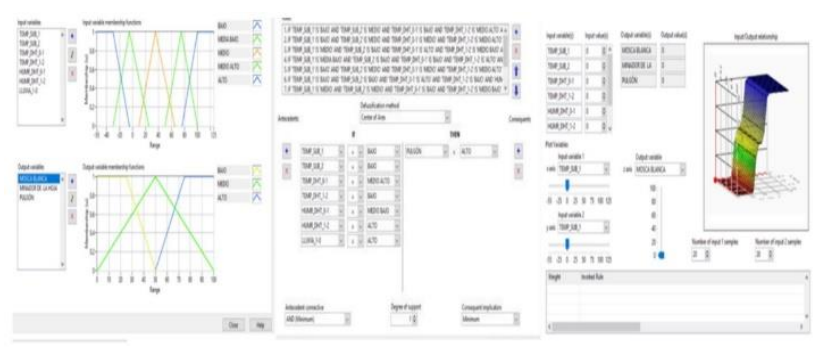

Figura 18 Modelo predictivo: Lógica difusa

Fuente: Elaboración Propia, 2019

\section{Evaluación en campo de cultivo}

Para la evaluación en campo de cultivo se contó con el apoyo total del Ing. Russell Escalante Rodríguez, productor de Chile Habanero del Estado de Campeche. Para tal fin, nos dio facilidades para la instalación del prototipo en un campo de cultivo ubicado en el poblado de Xcampeu con georreferencia 19.793162, 90.398320 (ver figura 19).

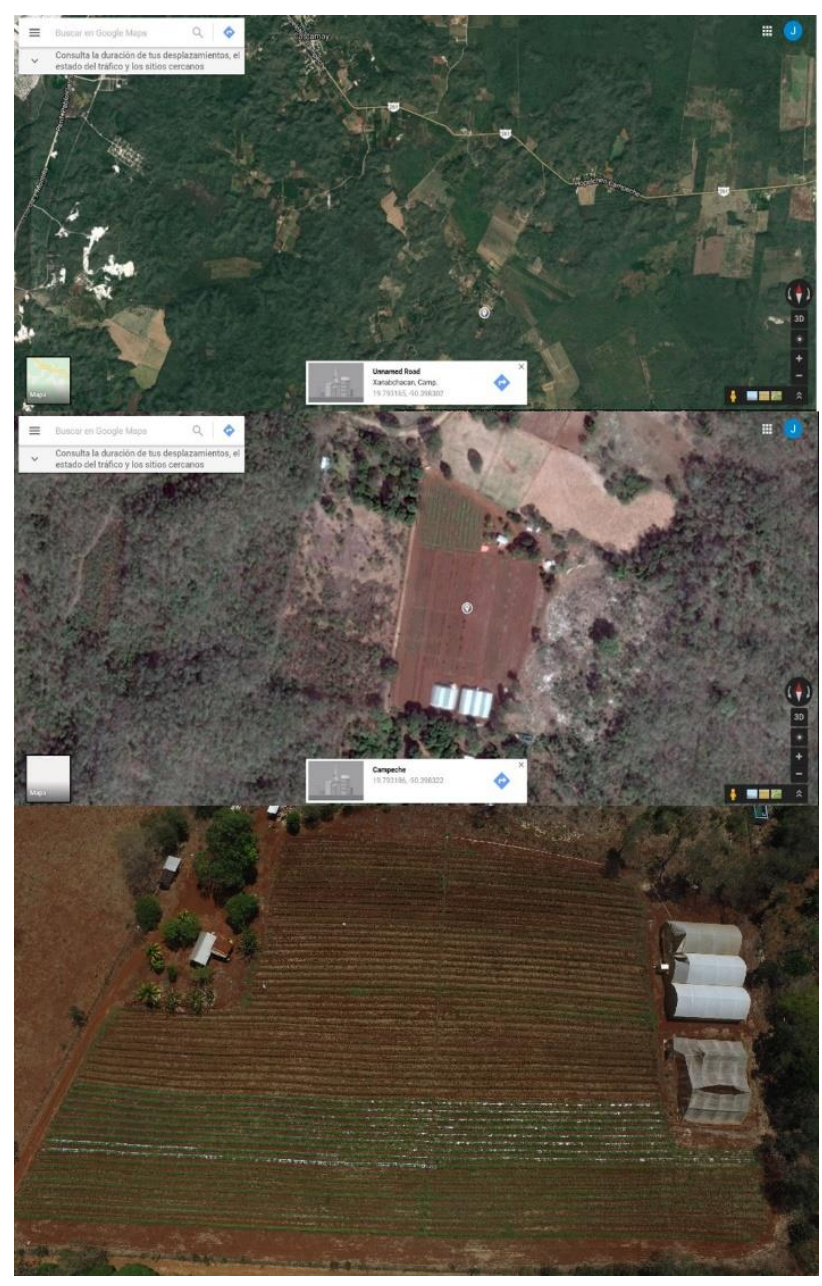

Figura 19. Ubicación campo de cultivo Fuente: Elaboración propia, 2019

Para la instalación del prototipo se evaluó la pertinencia del terreno debido a la cobertura del sensado y a la etapa en el ciclo de cultivo, dificultándose su instalación debido a lo compacto de la tierra a la escasa lluvia presentada en la temporada, en la imagen 20 se observa el prototipo instalado y funcionando. 


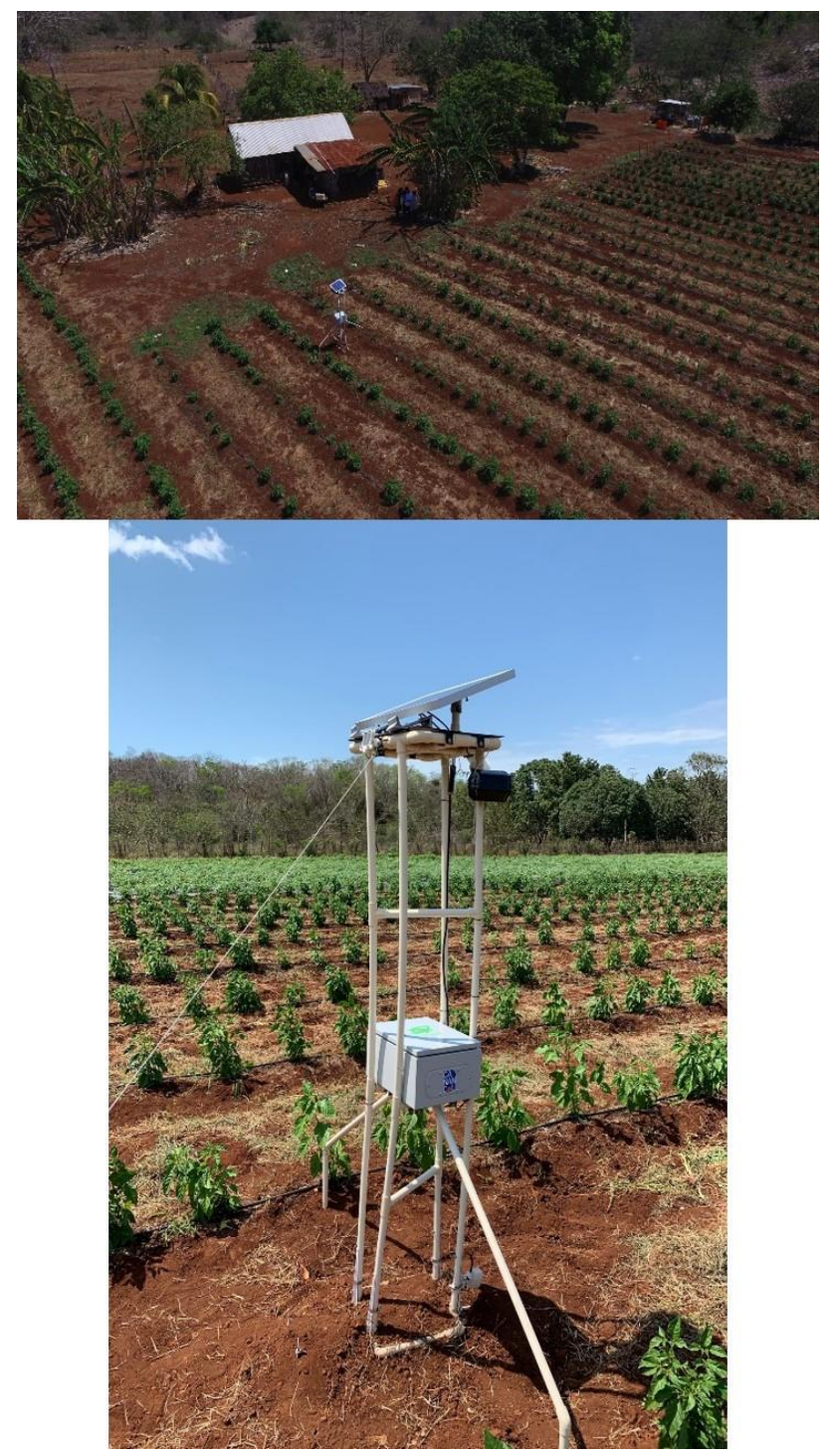

Figura 20 Instalación en campo de cultivo Fuente: Elaboración propia, 2019

Finalmente debido a la ubicación del cultivo, fue necesario colocar un repetidor de señal RF (figura 21) para alcanzar cobertura GSM y de este modo poder mandar la información recolectada hasta el servidor para su procesamiento y despliegue en la página web.

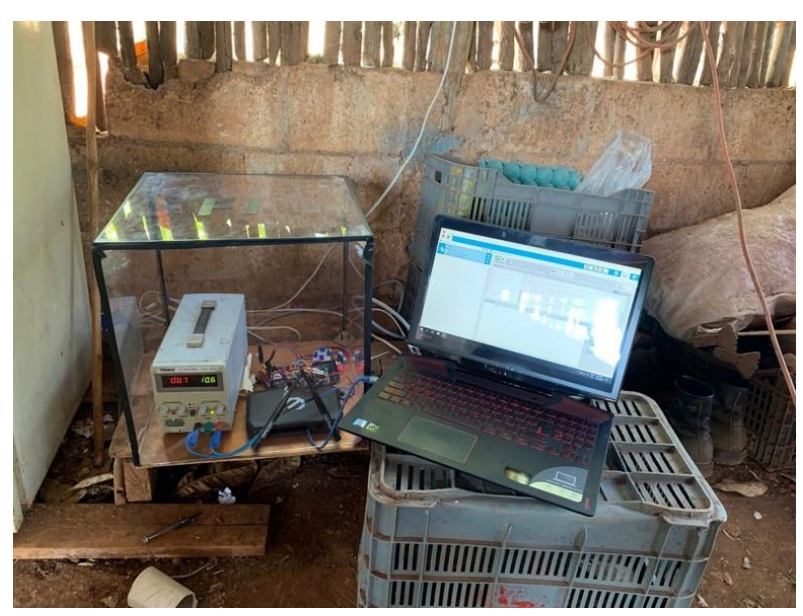

Figura 21. Repetidor RF y enlace GSM

Fuente: Elaboración Propia, 2019

\section{Resultados}

La evaluación del sistema planteado se llevó a cabo en tres cultivos experimentales diferentes de Chile Habanero variedad Kukulkán, con una extensión de 4 hectáreas y riego por goteo. De igual manera con la finalidad de no sesgar el estudio, fueron seleccionados para la validación dos Campos Experimentales de Centros de Investigación y un Campo de Cultivo de una Asociación Chilera. Para tal efecto, se realizaron convenios marco con los Centros de Investigación de "Colegio de Postgraduados Campus Campeche" y el "Instituto Nacional de Investigaciones Forestales, Agrícolas y Pecuarias", así como un acta de acuerdos con la "Asociación de Chileros de Campeche A.C." respectivamente. Para lograr obtener un comparativo, se solicitaron como datos de control los resultados de las perdidas obtenidas en el ciclo de cultivo inmediato anterior, costos de inversión y perdida, para posteriormente realizar el comparativo con respecto al ciclo de cultivo monitoreado con el sistema. Una vez concluido el ciclo de cultivo de los tres campos experimentales se obtienen los siguientes datos (Gráfico 1).

Porcentaje de cultivo perdido por ciclo

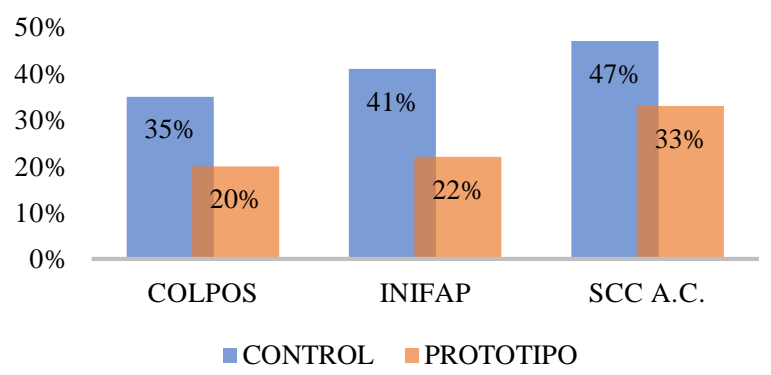

Gráfico 1. Resultados del sistema (pérdidas de cultivo) Fuente: Elaboración Propia, 2019

En lo que respecta a capital económico perdido por concepto de plagas y/o enfermedades, se debe tomar en cuenta que estos son en un rango de $\$ 360,000$ a $\$ 400,000$ pesos mexicanos, por tal motivo promediando el monto nos dan $\$ 380,000$ pesos mexicanos que un agricultor pierde por ciclo de cosecha de 4 hectáreas en cultivos de chile habanero, sin embargo como se muestra en el Gráfico 2, el capital perdido por el mismo concepto, se ve reducido con la implementación del prototipo, recuperando capital económico en un rango de $\$ 53,000$ a $\$ 72,000$ pesos mexicanos por ciclo de cultivo. 
Perdida económica por ciclo de cultivo

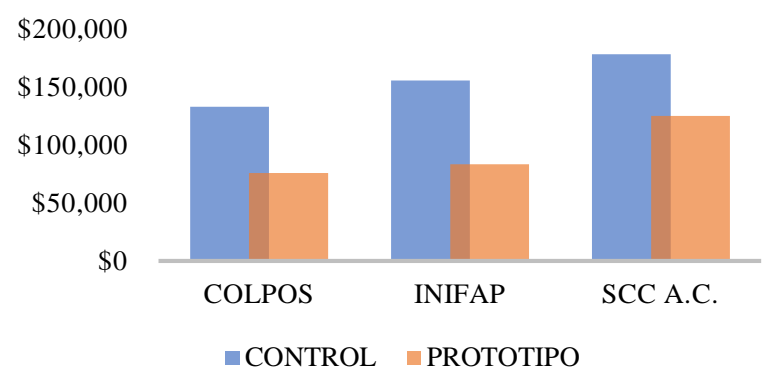

Gráfico 2. Resultados del sistema (pérdidas económicas) Fuente: Elaboración Propia, 2019

Dado lo anterior se logró validar el correcto funcionamiento del sistema de monitoreo remoto con un alcance de hasta 700 mts por RF, hasta llegar a la terminal principal GSM, ampliando de tal modo su conectividad.

Contemplando los costos por pérdida, el prototipo representa una inversión mínima del $3.98 \%$ al $6.57 \%$ recuperable de forma inmediata al término del primer ciclo de cultivo. Es importante señalar que en lo que respecta al equipo de cómputo necesario es a libertad del usuario ya que la interfaz gráfica de usuario se encuentra alojada en una página web sin necesidad de grandes requerimientos, por lo que los costos son de estructura y componentes necesarios. Igualmente, el sistema es desmontable por lo que, de requerirse en algún otro campo de cultivo, se puede desinstalar y montar en otra ubicación, por lo que no se generaría ningún costo extra, a reserva de requerirse repetidores extra por la lejanía de cobertura GSM.

En base a los datos suministrados por INIFAP y COLPOS, se desarrolló un sistema difuso para la predicción en base a la probabilidad de ataque de las plagas comunes en cultivos de chile habanero, siendo el pulgón, araña, gusano, minador de la hoja y mosca blanca. El sistema desarrollado fue del tipo MIMO (múltiples entradas múltiples salidas). Las entradas fueron los diferentes sensores colocados en la estructura in situ (como mencionó en el apartado de metodología), mientras que las funciones de pertenencia con sus respectivos rangos fueron determinadas por la bibliografía correspondiente a estudios biológicos realizados a las plagas por ambos Centros de Investigación.
Posteriormente las salidas, fueron los porcentajes de probabilidad de ser atacado el cultivo por alguna de las 6 plagas mencionadas anteriormente (6 variables de salida), para finalmente el usuario poder seleccionar el método de control óptimo para su cultivo, sea biológico o químico. Es importante señalar que la guía estratégica de control integrado de plagas mediante agentes biológicos y/o químicos, es ajustable a sus necesidades específicas, por lo que es flexible a cambios de parámetros dependiendo de la experiencia de cada productor.

Finalmente, además de la reducción de pérdidas de cultivos por plagas, se reduce un $32 \%$ la cantidad de agroquímicos usado en todo el ciclo de cultivo, ayudando de tal manera en disminuir los contaminantes para el manto acuífero de la región.

\section{Agradecimientos}

Agradecemos infinitamente por sus aportaciones en áreas de biología, así como a las pruebas realizadas a las siguientes instituciones:

\section{- Colegio de Postgraduados Campus Campeche (COLPOS). \\ - Instituto Nacional de Investigaciones Forestales, Agrícolas y Pecuarias Campus Campeche (INIFAP). \\ - $\quad$ Asociación de Chileros de Campeche.}

\section{Conclusiones}

En la implementación del presente prototipo se halló que uno de los principales motivos de la falta de tecnificación del campo es la baja aceptación de productores a las nuevas tecnologías y enraizamiento a técnicas de cultivo tradicional. De igual manera una barrera importante fue la lejanía de los terrenos de cultivo y la nula llegada de sistemas de telecomunicaciones.

Como se observa en el apartado de resultados los campos de cultivo con mejores resultados, fueron los administrados por los Centros de Investigación (reducción de hasta el $50 \%$ en pérdidas), esto es debido a que para lograr una correcta implementación del prototipo, se tiene que trabajar en perfecta sincronía con el personal agrónomo. 
Igualmente se observó una reducción significativa en el uso de agroquímicos en los campos de cultivo, usando un $68 \%$ del total que se esparce en un ciclo de cultivo, lo que a su vez se refleja en la disminución de contaminantes para el manto acuífero de la región.

Finalmente para el caso de cultivos de Chile Habanero, mediante un sistema de monitoreo remoto, integrando en una Interfaz Gráfica de Usuario implementada en una Web Page amigable a la vista e intuitiva, se logró anticipar el ataque potencial las principales plagas que inciden en él, previniendo de tal modo las enfermedades en los cultivos; brindando a los productores una guía estratégica de control sustentable de plagas mediante agentes biológicos y/o químicos, ajustable a sus necesidades específicas.

\section{Referencias}

Arias, D. M., Roldan, M. V. H. P., \& Vélez, M. U. (2019). USO DEL ÍNDICE NORMALIZADO DE VEGETACIÓN PARA LA ELABORACIÓN DE PLANOS DE CULTIVO. Opuntia Brava, 11(2), 261-265.

Borges-Gómez, L., Cervantes Cárdenas, L., Ruiz Novelo, J., Soria Fregoso, M., Reyes Oregel, V., \& Villanueva Couoh, E. (2010). Capsaicinoides en chile habanero (Capsicum chinense Jacq.) bajo diferentes condiciones de humedad y nutrición. Terra latinoamericana, 28(1), 35-41.

Bosland, P. W. (1996). Capsicums: Innovative uses of an ancient crop. Progress in new crops. ASHS Press, Arlington, VA, 479-487.

Cauich, I. C., Ascencio, F. J., \& Cen, F. A. C. BENEFICIO ECONÓMICO DE LA PRODUCCIÓN DE CHILE HABANERO (Capsicum chinense) EN EL MUNICIPIO DE HALACHÓ, YUCATÁN.

Cázares Sánchez, E., Ramírez Vallejo, P., Castillo González, F., Soto Hernández, R. M., Rodríguez González, M. T., \& Chávez Servia, J. L. (2005). Capsaicinoides y preferencia de uso en diferentes morfotipos de chile (Capsicum annuum L.) del centro- oriente de Yucatán. Agrociencia, 39(6).
Cuyo, Q., \& Pedro, J. (2019). Automatización y monitoreo de un túnel para el secado de la incendios forestales e invasión de retamo espinoso en los Cerros Orientales de Bogotá. Acta Biológica Colombiana, 24(1), 1-12.

INIFAP (Instituto Nacional de Investigaciones Forestales Agrícolas y Pecuarias). (2017). Fertigación de chile habanero en suelos pedregosos de Yucatán. Secretaría de Agricultura, Ganadería, Desarrollo Rural, Pesca y Alimentación. México, D.F.

Kú, V. M. I., \& Gary, J. D. (2004). Asociación de la diversidad genética de los cultivos de la milpa con los sistemas agrícolas y factores socioeconómicos en una comunidad de Yucatán.

Lee, C. C. (1990). Fuzzy logic in control systems: fuzzy logic controller. II. IEEE Transactions on systems, man, and cybernetics, 20(2), 419-435.

LONG-SOLÍS, J., Alvarez, M., \& Camarena, A. (1998). El placer del chile. Editorial Clio. México.

Manrique, J. M. T., Martínez, E., \& Méndez, G. M. (2014). Análisis comparativo de la sustentabilidad de dos unidades productivas de Chile Habanero convencionales y dos unidades productivas orgánicas en Yucatán, México. Observatorio de la Economía Latinoamericana, (194).

Moreno Toro, A., \& Salgado Polo, E. (2019). Análisis de cultivo de maíz para ensilaje utilizando imágenes desde un UAV.

Moreno, A., \& Arnaldo, W. (2019). Automatización de un Sistema Multiplataforma de Control y Monitoreo de Riego Tecnificado para el Distrito de los Palos en el Año 2017.

Navarro, L. A. A., López, J. F. J., López, D. J. P., López, R. L., Hernández, M. B. R., Ramírez, M. J. A. R., ... \& Sánchez, A. M. ESTUDIO PARA DETERMINAR ZONAS DE ALTA POTENCIALIDAD DEL CULTIVO DEL CHILE HABANERO (Capsicum chinense Jacq.) EN EL ESTADO DE TABASCO. 
Ocampo-zuleta, K. (2019). Modelo descriptivo de restauración ecológica en zonas afectadas por producción de la harina de maca en la localidad de Junín.

Ramírez, H., Amado-Ramírez, C., BenavidesMendoza, A., Robledo- Torres, V., \& MartínezOsorio, A. (2010). Prohexadiona-Ca, AG3, ANOXA y BA modifican indicadores fisiológicos y bioquímicos en chile Mirador. Revista Chapingo. Serie horticultura, 16(2), 8389.

Razavi, B. (2012). RF microelectronics. Pearson Education.

Ross, T. J. (2004). Fuzzy logic with engineering applications (Vol. 2). New York: Wiley.

Ruiz, B. R. (2009). Potencial productivo y limitante para la producción de chile habanero (Capsicum chinense Jacq.) en la zona centro del estado de Veracruz (Doctoral dissertation, Tesis Doctoral, Campus Veracruz. Colegio de Postgraduados, Manlio Fabio Altamirano, Veracruz).

Ruiz-Lau, N., Medina-Lara, F., \& MartínezEstévez, M. (2011). El chile habanero: su origen y usos. Revista ciencia.

SAGARPA, S. (2007). Secretaria de agricultura, ganadería, desarrollo rural, pesca y alimentación; servicio de información agroalimentaria y pesquera.

SAGARPA, S. (2018). Secretaria de agricultura, ganadería, desarrollo rural, pesca y alimentación; servicio de información agroalimentaria y pesquera.

Salazar-Jara, F. I., \& Juarez-Lopez, P. (2013). Requerimiento macronutrimental en plantas de chile (Capsicum annuum L.).

Schmuller, J. (2000). Aprendiendo UML en 24 horas. Pearson educación.

Stetzler, T. D., Post, I. G., Havens, J. H., \& Koyama, M. (1995). A 2.7-4.5 V single chip GSM transceiver RF integrated circuit. IEEE Journal of Solid-State Circuits, 30(12), 14211429.
Villa Castorena, M., Catalán Valencia, E. A., Inzunza Ibarra, M. A., Román López, A., Macias Rodríguez, H., \& Cabrera Rodarte, D. (2014). Producción hidropónica de chile habanero en invernadero. 